\title{
RELACIÓN ENTRE DOPPLER DE LA ARTERIA UMBILCAL, INVASIÓN MICROBIANA DE LA CAVIDAD AMNIÓTICA, FUNISITIS Y RESULTADO ADVERSO NEONATAL EN LA ROTURA PREMATURA DE MEMBRANAS DE PREIÉRMINO
}

\author{
Alfredo Ovalle S. ${ }^{1}$, Jorge Figueroa P. ${ }^{1}$, Ricardo Gómez $M .^{2}, M$. Angélica Martínez $T^{3}$, \\ Miriam Ocaranza B. ${ }^{1}$, Elena Kakarieka W. ${ }^{4}$, Ariel Fuentes ${ }^{1}{ }^{1}$, Mercedes Ruiz . $^{1}$

\begin{abstract}
${ }^{1}$ Servicio y Departamento de Obstetricia, Ginecología y Neonatología, Hospital San Borja Arriarán, Facultad de Medicina, Universidad de Chile. ${ }^{2}$ Centro de Diagnóstico e Investigaciones Perinatales (CEDIP), Hospital Dr Sótero del Río. ${ }^{3}$ Programa de Microbiología, ICBM, Facultad de Medicina, Universidad de Chile. ${ }^{4}$ Servicio de Anatomía Patológica, Hospital San Borja Arriarán.
\end{abstract}

\section{RESUMEN}

Objetivo: Evaluar la utilidad del Doppler de la arteria umbilical (AU) para predecir invasión microbiana de la cavidad amniótica (IMCA), funisitis y resultado adverso neonatal (RA) en pacientes con rotura prematura de membranas de pretérmino (RPMPT). Métodos: Se estudian 80 embarazadas entre 24 y 34 semanas de gestación con diagnóstico de rotura prematura de membranas. Se excluyeron embarazadas con condiciones materno-fetales severas que pudiesen alterar el resultado perinatal. Todas tuvieron ultrasonografía para biometría fetal y Doppler de la arteria umbilical dentro de una semana del nacimiento y microbiología de líquido amniótico. Se creó una variable compuesta que incluyó morbilidad neonatal severa, secuelas o muerte neonatal. Las pacientes recibieron antibióticos, esteroides y manejo expectante hasta las 35 semanas. IMCA se definió por el cultivo positivo del líquido amniótico; funisitis por la presencia de leucocitos polimorfonucleares en la pared de los vasos umbilicales o gelatina de Warthon. Se usaron análisis de curva ROC y tablas de contingencia para el cálculo estadístico. Resultados: Se incluyeron 68 pacientes. El RA compuesto se presentó en 19,4\%. Los fetos que desarrollaron RA tuvieron relación S/D de $A U$, significativamente más alta que los fetos con resultado bueno (RB) $(65,6 \pm 30,9$ vs $30,0 \pm 20,4$ $p<0,001)$, así como también más alta proporción de valores de la relación $S / D$ de la AU sobre el percentil $90(30,8 \%$ vs $0 \%$, respectivamente, $p<0,0001)$. No hubo diferencias en la relación $S / D$ de la $A U$ en los grupos con y sin IMCA y con o sin funisitis. Fetos con relación $S / D$ de la AU con percentil $>41$ tuvieron significativo más alto riesgo de RA que fetos con percentil < 41 (odds ratio: 15,7; 95\% Cl 2,73-118; $\mathrm{p}<0,001$ ), con sensibilidad de $85 \%$, tasa de falso-positivo de $56 \%$, especificidad de $74 \%$ y falso negativo de $5 \%$. Conclusiones: En la RPMPT, la relación S/D de la $\mathrm{AU}$, predice el resultado neonatal adverso; este procedimiento no detecta IMCA y funisitis.

\section{PALABRAS CLAVES: Velocimetría Doppler, arteria umbilical, rotura prematura de membranas de pretérmino, pronóstico perinatal adverso, funisitis}

\section{SUMMARY}

Objective: To evaluate the usefulness of Doppler velocimetry of umbilical artery in the prediction of microbial invasion of the amniotic cavity (MIAC), funisitis and adverse neonatal outcome in patients with 
preterm PROM. Methods: 80 patients with preterm PROM between 24 and 34 weeks were invited to participate in this study. Exclusion criteria were fetal and maternal conditions that may influence perinatal outcome. All patients underwent obstetrical ultrasound, biophysical profile and Doppler velocimetry of the umbilical artery (UA) systole/diastole (S/D) measurements within 1 week of delivery. An amniocentesis was performed at admission for amniotic fluid microbiological assessment. MIAC was defined as the presence of a positive amniotic fluid culture. Patients received antibiotics, steroids and were managed expectantly until 35 weeks. A composite variable including severe neonatal morbidity, sequelae or death was created. Funisitis was diagnosed in the presence of polymorphonuclear leukocyte infiltration into the umbilical vessel walls or Wharton jelly. Statistics were performed using ROC curve analysis and contingency tables. Results: 68 patients were included. Composite neonatal morbidity was present in $19.4 \%$. Fetuses that subsequently developed adverse neonatal outcome had a significantly higher mean UA S/D percentile for gestational age than fetuses without subsequent adverse neonatal outcome $(65.6 \pm 30.9$ vs $30.0 \pm 20.4 ; p<0.001)$ as well as a higher proportion of UA S/D values above the 90th percentile (30.8\% vs $0 \%$, respectively, $p<0.0001)$. There were no differences in the mean UA S/D values between fetuses with and without MIAC and those with and without funisitis. Fetuses with UA S/D percentile $>41$ had a significantly higher risk of adverse outcome than fetuses with percentile < 41 (odds ratio: $15.7 ; 95 \% \mathrm{Cl} 2.73-118 ; \mathrm{p}<0.001$ ) with a sensitivity: $85 \%$, false-positive rate: $56 \%$, specificity: $74 \%$, and false-negative: $5 \%$ ). Conclusions: Among patients with preterm PROM, umbilical artery Doppler velocimetry was significantly associated with adverse neonatal outcome, but it was not related to microbial invasion of the amniotic cavity or funisitis.

\section{KEY WORDS: Doppler velocimetry, umbilical artery, preterm premature rupture of membranes, adverse neonatal outcome, funisitis}

\section{INTRODUCCIÓN}

La rotura prematura de membranas de pretérmino (RPMPT), se presenta en un tercio de los partos prematuros y antes de las 32 semanas de gestación se asocia con alta morbimortalidad perinatal $(1,2)$. Estos riesgos han sido relacionados con invasión microbiana de la cavidad amniótica (IMCA) (3-5) y en menor grado con infección cérvicovaginal (6). Las consecuencias de la infección intrauterina (corioamnionitis clínica, corioamnionitis histológica, funisitis y aumento de citoquinas intraamnióticas) en el niño se asocian con sepsis, bronconeumonía, enterocolitis necrotizante (3-5), con daños neurológicos (leucomalacia periventricular, hemorragia intraventricular, parálisis cerebral) y enfermedad crónica pulmonar (710). El pronóstico adverso del neonato se ha correlacionado particularmente con la funisitis, presente en $61 \%$ de las embarazadas con IMCA (11). La funisitis se relaciona con muerte perinatal, infección, asfixia severa, ingreso frecuente del neonato a la Unidad de Cuidado Intensivo y parálisis cerebral $(11,12)$.

La medición de la velocimetría Doppler de la arteria uterina $(A U)$, método diagnóstico no invasivo, eficiente y rápido, ha resultado útil para predecir complicaciones perinatales y admisión a la Unidad de Cuidado Intensivo en fetos con retraso del crecimiento intrauterino $(13,14)$. En cambio en la RPMPT, no se ha logrado relacionar con
IMCA, corioamnionitis clínica, inflamación placentaria e infección perinatal, características analizadas individualmente (15-18).

Es posible que un resultado neonatal compuesto (19), que incluya síndrome de dificultad respiratoria, asfixia severa, sepsis, bronconeumonía, hemorragia intraventricular, secuelas graves como leucomalacia periventricular, displasia broncopulmonar y muerte pueda mejorar el rendimiento del Doppler en estos casos. De este modo se podría predecir el resultado adverso neonatal y decidir la interrupción de la gestación.

Los objetivos de este estudio fueron evaluar la utilidad de la velocimetría Doppler de la arteria umbilical para predecir IMCA, funisitis y resultado neonatal adverso en pacientes con RPMPT y explorar los diferentes puntos de corte de la curva de valores de la relación sístole diástole (S/D) para optimizar la detección de los fetos con riesgo.

\section{MATERIAL Y MÉTODO}

Criterios de selección. Entre abril de 1997 y noviembre de 2003, embarazadas entre 24 y 34 semanas de gestación con el diagnóstico de rotura prematura de membranas (RPM) fueron invitadas a participar en este trabajo. La Figura 1 muestra el diagrama de flujo que describe el proceso de enrolamiento de pacientes. Se incorporaron sólo pacientes admitidas durante la mañana de 


\begin{tabular}{lr}
\hline Pacientes & $\mathbf{n}$ \\
\hline Seleccionadas & 428 \\
No seleccionadas & 348 \\
Enroladas & 80 \\
Excluidas & 12 \\
Aprobaron criterios de selección & 68 \\
\hline
\end{tabular}

Figura 1. Diagrama de flujo de las pacientes seleccionadas.

los días de semana por razones técnicas del estudio microbiológico. La rotura de membranas fue clínicamente documentada visualizando pérdida evidente de líquido amniótico a través del orificio cervical con espéculo estéril y/o empleando la prueba de la nitrazina. El Comité de Ética del establecimiento aprobó la realización de este trabajo. Se obtuvo consentimiento informado de cada paciente participante. La edad gestacional se determinó por la historia menstrual cuando fue confiable, examen pélvico en el primer trimestre de acuerdo a la extensión de la amenorrea o biometría fetal ultrasonográfica antes de las 24 semanas. Se hizo evaluación de la dilatación cervical y se tomaron muestras cervicales y vaginales para cultivo durante el examen con espéculo. No se hizo examen vaginal digital durante la admisión. Todas las pacientes tuvieron ultrasonografía obstétrica para biometría fetal, relación S/D de la arteria umbilical y evaluación de líquido amniótico.

Criterios de exclusión. Se excluyeron a las embarazadas en trabajo de parto, uso de antibióticos durante los 30 días previos a la admisión, última medición de Doppler realizada dos semanas antes del nacimiento, hemorragia genital significativa, desprendimiento prematuro de placenta, anomalía congénita o muerte fetal, anomalías uterinas, sufrimiento fetal, presencia de dispositivo intrauterino, gestación múltiple, infección ovular clínica (corioamnionitis clínica), infección extrauterina incluyendo pielonefritis, enfermedad médica materna que determinase interrupción de la gestación y restricción del crecimiento fetal intrauterino (< del percentil 10 para la edad gestacional) (20).

Estudio microbiológico. Muestras cervicales, vaginales y de líquido amniótico se obtuvieron para estudio microbiológico. Una tórula de la muestra vaginal se usó para la preparación de la placa para la tinción de Gram y luego se colocó en medio de transporte de Stuart, destinado para la recuperación de $S$. agalactiae, de Candida especies y de otras bacterias aeróbicas y anaeróbicas facultativas. Una segunda tórula endocervical fue inoculada en medio de transporte SP (sacarosa fosfato) y utilizada para preparación de muestra húmeda, cultivo de mycoplasmas urogenitales y diagnóstico de $C$. trachomatis por amplificación de DNA (PCR) $(21,22)$. La muestra húmeda se usó para recuento de leucocitos polimorfonucleares al exámen microscópico al fresco y para el diagnóstico de $T$. vaginalis (6).

La muestra de líquido amniótico, obtenida por amniocentesis transabdominal, fue inoculada en medios tioglicolato prerreducido y en 2 SP. La muestra restante se transportó al laboratorio en una jeringa con cubierta estéril inmediatamente después de su recolección. Los cultivos para bacterias aeróbicas y anaeróbicas facultativas y estrictas así como para Mycoplasmas urogenitales fueron realizados de acuerdo a métodos previamente descritos (23-25).

El diagnóstico microbiológico de vaginosis bacteriana fue efectuado mediante la evaluación de la tinción de Gram del flujo vaginal según el método propuesto por Nugent y cols (27). Invasión microbiana de la cavidad amniótica fue definida por el cultivo positivo del líquido amniótico (28). Infección cérvicovaginal (ICV) se consideró según los siguientes criterios: a) presencia de vaginosis bacteriana 0 b) cultivo positivo para bacteria patógena o bacteria facultativa en cérvix o vagina (excepto por Lactobacillus) asociado con incremento de leucocitos polimorfonucleares sobre 10 por campo al examen microscópico directo (400x) $(6,29)$.

Manejo de las pacientes. El líquido amniótico se usó para estudio microbiológico y evaluación de la madurez pulmonar. Todas las pacientes recibieron 2 dosis de betametasona $12 \mathrm{mg}$ IM cada $24 \mathrm{~h}$. Se administraron los siguientes antimicrobianos: clindamicina $600 \mathrm{mg}$ intravenosamente cada 8 horas por 48 horas y gentamicina $4 \mathrm{mg} / \mathrm{kg} /$ día intravenosa en una dosis por 48 horas. Luego se continuó con clindamicina $300 \mathrm{mg}$ cada 6 horas oral por 5 días y gentamicina $4 \mathrm{mg} / \mathrm{kg} / \mathrm{día}$ intramuscular en una dosis por 5 días. La utilidad de este esquema está demostrada en publicación anterior de estos autores (30). Tocolisis no se usó en este estudio.

Se manejaron las pacientes en forma expectante hasta las 35 semanas completas. Para la vigilancia fetal se realizó monitorización electrónica de la frecuencia cardíaca fetal, perfil bíofísico fetal, hemograma, proteína $C$ reactiva y cultivos de líquido amniótico y endocervical semanales. El parto se indicó con el desarrollo de alguna de las siguientes condiciones: infección ovular clínica 
(corioamnionitis clínica), cultivo de líquido amniótico positivo para $S$. agalactiae, N. gonorrhoeae, $H$. influenzae, E. coli o F. nucleatum, sufrimiento fetal, prolapso de cordón y desprendimiento prematuro de placenta.

Infección ovular clínica o corioamnionitis clínica fue definida según los criterios de Gibbs y cols. El diagnóstico requirió elevación de la temperatura axilar a $37,8^{\circ}$ C o más, acompañado de dos de los siguientes criterios: hipersensibilidad uterina, secreción vaginal fétida, taquicardia materna y fetal y leucocitos > de 15.000 células $/ \mathrm{mm}^{3}$ (31). Se definió endometritis puerperal como la elevación de la temperatura axilar a $38^{\circ} \mathrm{C}$ o más en dos tomas (separadas por más de 4 horas), con sensibilidad uterina y loquios turbios de mal olor y sin otra causa aparente de infección (31). A las pacientes con infección ovular clínica se les indicó el parto con administración de clindamicina-gentamicina. Las mujeres con endometritis puerperal recibieron similar asociación de antimicrobianos.

Velocimetría Doppler. Se evaluó la onda de velocidad de flujo de la arteria umbilical (AU), con un ecógrafo ATL HDI 3500 con transductor multifrecuencia (3.5-5.0 MHz). La medición la hicieron dos ultrasonografistas (J.F. y M.O.). Con la paciente en semi-Fowler se obtuvo una señal vascular de AU en asa libre de cordón y en ausencia de movimientos respiratorios tanto maternos como fetales. Se obtuvo de esta forma, al menos cinco ondas de velocidad de flujo pulsátil en las que se midió la relación $S / D$ (desde el máximo peak sistólico al mínimo peak diastólico) la que fue calculada automáticamente por el software del computador del ecógrafo. Los valores de la relación S/D así obtenidos se convirtieron en percentiles para cada edad gestacional (32). La medición S/D se realizó cada 72 horas hasta el momento del parto en las pacientes con IMCA y semanalmente en aquéllas sin IMCA. Para el análisis de los resultados sólo se consideró la medición más cercana al parto.

Complicaciones neonatales y manejo. Se advirtió a los neonatólogos del estado microbiológico de la cavidad amniótica y del tratamiento antibiótico recibido. Todos los neonatos bajo 2.000 $\mathrm{g}$ recibieron ampicilina y amikacina endovenosa, luego de tomarse los siguientes exámenes: hemocultivos, proteína $C$ reactiva, hemograma y cultivos microbiológicos periféricos y de contenido gástrico. Los antibióticos se descontinuaron en ausencia de elementos clínicos de infección y con exámenes de laboratorio negativos.

Las siguientes complicaciones de la primera semana de vida se analizaron: Sepsis neonatal se diagnosticó por la presencia de cultivos positivos de sangre o cerebroespinal o cuando el niño presentó cuadro multisistémico clínicamente compatible con el diagnóstico. Bronconeumonia se diagnosticó por cuadro clínico y radiológico compatible. Síndrome de dificultad respiratoria (SDR) se definió como el cuadro respiratorio con quejido y retracción, con requerimientos de $\mathrm{O}_{2}$ aumentado $\left(\mathrm{FiO}_{2}>0,4\right)$, gases arteriales alterados y documentado con radiología compatible. Enterocolitis necrotizante se diagnosticó en presencia de distensión abdominal, intolerancia alimentaria 24 horas o más, con clara evidencia radiológica de aire intramural, perforación, síndrome meconial o específicos hallazgos de enterocolitis necrotizante en la cirugía o autopsia. Hemorragia intraventricular diagnosticada por el examen ultrasonográfico de la cabeza del neonato; se admitieron lesiones grado III y IV (hemorragias intraventriculares severas) (33). Asfixia severa se consideró cuando el neonato presentó test de Apgar de 3 puntos o menos a los 5 minutos.

Se creó un resultado neonatal compuesto incluyendo algunas de las siguientes condiciones (síndrome de dificultad respiratoria, asfixia severa, sepsis, bronconeumonía y hemorragia intraventricular severa) seria secuela neonatal (leucomalacia periventricular y displasia broncopulmonar) y muerte neonatal. Leucomalacia periventricular se diagnosticó en presencia de lesiones quísticas dentro de la sustancia blanca periventricular o anormal persistencia de ecogenicidad periventricular aumentada. Displasia broncopulmonar se diagnosticó si el neonato requirió oxígeno y terapia ventilatoria por más de 28 días durante los dos primeros meses de vida, si tuvo típicos cambios radiográficos, o si tuvo displasia del árbol broncopulmonar a la autopsia. Es posible que la combinación de esta morbilidades (19) pueda mejorar el rendimiento del Doppler en estos casos.

Estudio histológico placentario. Las muestras de tejido se obtuvieron del cordón umbilical, de la zona más gruesa del plato central y de las membranas extraplacentarias, se fijaron en formalina al $10 \%$ por 48 horas y se incluyeron en parafina. Se obtuvieron secciones de tejido de 3 a 5 micrones de espesor y se tiñeron con hematoxilina y eosina (H \& E) y ácido periódico de Schiff (PAS). El exámen histopatológico fue realizado por un sólo patólogo (E.K.). Los hallazgos histopatológicos se clasificaron en: 1) marcadores histológicos de infecciones ascendentes agudas 2) lesiones vasculares fetales: 
1) Marcadores histológicos de infección intrauterina ascendente incluye corioamnionitis (presencia de leucocitos polimorfonucleares que infiltran las membranas fetales, amnios y corion con o sin necrosis), funisitis (inflamación aguda del cordón umbilical caracterizado por infiltración de leucocitos polimorfonucleares en la pared de los vasos umbilicales o gelatina de Warthon) y perivellositis o intervellositis (infiltrado inflamatorio polimorfonuclear o mononuclear en el espacio intervellositario) (34-37).

2) Lesiones vasculares fetales pueden ser inducidas por hipoxia e incluye endoarteritis obliterante (edema y proliferación de las células de la íntima con engrosamiento y duplicación de la membrana basal con posible oclusión de los vasos fetales), endovasculitis hemorrágica (degeneración del endotelio de los vasos fetales y trombosis), corangiosis (aumento de los vasos de la vellosidad definido por la observación de 10 o más vasos en 10 o más vellosidades, en 10 o más campos al examen microscópico 400x) (34-37). Hiperplasia del trofoblasto: proliferación aumentada del trofoblasto caracterizada por crecimiento velloso y aparición de nudos y yemas sinciciales con gran cantidad de núcleos, que crecen en la superficie vellosa. La existencia de hipoxia induce la proliferación sincicial. Se asocia con hipertensión arterial, enfermedades maternas, anemia, sufrimiento fetal e infección ovular (34-38). Trombosis intervellositaria: lesión formada por trombos laminados, antiguos y recientes, en el centro del espacio intervelloso, debida a daño vellositario y turbulencias del flujo sanguíneo materno. Infarto: área localizada de necrosis vellosa isquémica, asociada con enfermedad vascular úteroplacentaria. Hematoma retroplacentario: hematoma que se encuentra entre el plato basal de la placenta y la pared uterina y los separa. Los efectos pueden ser más serios en presencia de severa enfermedad arterial úteroplacentaria (34-38).

Expresión de resultados. Para evaluar la utilidad del Doppler en pacientes con RPMPT, se midió la histología de la placenta, la infección (IMCA, cérvicovaginal) y el resultado adverso (RA) neonatal (alguna morbilidad neonatal severa, secuelas y muerte). La relación $S / D$ de la $A U$ se midió y se expresó en percentil en los grupos con y sin RA neonatal, con y $\sin$ IMCA y con y $\sin$ funisitis. Se exploraron los diferentes puntos de corte de la curva de valores de la última medición S/D para optimizar la detección de los fetos con riesgo.

Análisis estadístico de los datos. Se usó
Test de chi cuadrado y exacto de Fisher en la comparación de proporciones; Test t o de Wilcoxon en la comparación de variables continuas y análisis de varianza según correspondiese. Para evaluar la distribución normal de las variables continuas se empleó el Test de Kolmogorov. Se usó análisis de curva ROC (receiver operating characteristic) como predictor independiente de resultado neonatal adverso. Un valor de $p<0,05$ fue considerado significativo.

\section{RESULTADOS}

Características de la población y exclusiones: 80 pacientes fueron enroladas en este estudio. Doce mujeres fueron excluidas: seis casos con restricción del crecimiento fetal $(<$ percentil 10), un caso con síndrome de Down y restricción del crecimiento fetal, dos casos que finalmente tuvieron las membranas íntegras, un caso con falla en la obtención de líquido amniótico, un caso con edad gestacional < de 24 semanas corregida posteriormente y un caso letal de malformación fetal.

68 pacientes cumplieron con los criterios de inclusión. La Tabla I describe las características de la población en relación al resultado neonatal. En el grupo de pacientes con resultado neonatal adverso (RA) en comparación con el grupo con resultado bueno (RB), la edad gestacional al ingreso $(27,1$ semanas vs 30,9 semanas; $p<0,0001)$ $y$ el peso neonatal $(1.208 \mathrm{~g}$ vs $2.010 \mathrm{~g} ; \mathrm{p}<0,0001)$ fueron significativamente menores.

La admisión a la $\mathrm{UCl}$ fue más frecuente en neonatos con RA (100\% [13/13]) que con RB $(61,1 \%[33 / 54] p<0,01)$ Ninguno de los recién nacidos tuvo retraso del crecimiento fetal $(<$ percentil 10).

En los casos con cultivo de líquido amniótico positivo para $S$. agalactiae, $H$. influenzae, $F$. nucleatum y $N$. gonorrhoeae (12 pacientes) y en los casos con corioamnionitis clínica (7 pacientes) el parto se desencadenó espontáneamente en todos los casos antes de que fuese necesaria cualquier intervención.

14 neonatos tuvieron RA (se excluyó una muerte debida a trauma en el parto: recién nacido con $739 \mathrm{~g}$, parto vaginal en presentación podálica, con hematoma hepático y muerte intraparto por shock). El resultado compuesto se presentó en 19,4\% (13/ 67) de los neonatos: 1) neonato con sepsis y bronconeumonía, 2) neonato con SDR, sepsis, bronconeumonía, asfixia severa y muerte, 3) neonato con HIV, bronconeumonía y leucomalacia 
Tabla I

POBLACIÓN EN ESTUDIO

\begin{tabular}{|c|c|c|c|}
\hline & $\begin{array}{c}\text { Resultado adverso* } \\
n=13\end{array}$ & $\begin{array}{l}\text { Resultado bueno } \\
n=54\end{array}$ & Valor $p$ \\
\hline \multicolumn{4}{|l|}{ Edad materna (años) } \\
\hline Media (DS) & $28,7(7,7)$ & $29,7(6,9)$ & \multirow[t]{2}{*}{ NS } \\
\hline Rango & $18-43$ & $17-40$ & \\
\hline \multicolumn{4}{|l|}{ Paridad } \\
\hline Nulípara & $3(23,1 \%)$ & $14(25,9 \%)$ & \multirow[t]{2}{*}{ NS } \\
\hline Multípara & $10(76,9 \%)$ & $40(74,1 \%)$ & \\
\hline \multicolumn{4}{|l|}{ Edad gestacional admisión (semanas) } \\
\hline Media (DS) & $27,1(1,6)$ & $30,9(2,5)$ & \multirow[t]{2}{*}{$<0,0001$} \\
\hline Rango & $25,0-31,1$ & $25-34$ & \\
\hline \multicolumn{4}{|l|}{ Modo de nacimiento } \\
\hline Vaginal & $5(38,5 \%)$ & $28(51,9 \%)$ & \multirow[t]{2}{*}{ NS } \\
\hline Cesárea & $8(61,5 \%)$ & $26(48,1 \%)$ & \\
\hline \multicolumn{4}{|l|}{ Intervalo admisión-parto (días) } \\
\hline Media (DS) & $6,9(8,5)$ & $9,5(8,8)$ & \multirow[t]{2}{*}{ NS } \\
\hline Mediana (rango) & $4,0(0-31)$ & $9,0(1-39)$ & \\
\hline Admisión a unidad cuidado intensivo* & $13(100 \%)$ & $33(61,1 \%)$ & $<0,01$ \\
\hline \multicolumn{4}{|l|}{ Peso nacimiento (g) } \\
\hline Media (SD) & $1.208(323)$ & $2.010(501)$ & \multirow[t]{2}{*}{$<0,0001$} \\
\hline Rango & $800-2.150$ & $888-2.970$ & \\
\hline
\end{tabular}

*Se excluye una muerte por trauma en el parto.

periventricular, 4) neonato con asfixia severa y displasia broncopulmonar, 5) neonato con HIV y leucomalacia periventricular, 6) neonato con HIV, sepsis, bronconeumonía y leucomalacia periventricular, 7) neonato con HIV, 8) neonato con asfixia severa y displasia broncopulmonar, 9) neonato con asfixia severa, SDR, HIV y muerte, 10) neonato con extensa bronconeumonía bilateral, 11) neonato

Tabla II

\section{RESULTADOS NEONATALES ADVERSOS}

\begin{tabular}{lrr}
\hline & $n$ & \multicolumn{1}{c}{$\%$} \\
\hline Síndrome de dificultad respiratoria & 3 & 4,5 \\
Asfixia severa & 6 & 9,0 \\
Hemorragia intraventricular severa & 5 & 7,5 \\
Infección neonatal $^{\text {Secuela severa* }}$ & 7 & 10,4 \\
Muerte** $^{\star *}$ & 6 & 9,0 \\
Resultado neonatal compuesto $^{* * *}$ & 5 & 7,5 \\
& 13 & 19,4 \\
\hline
\end{tabular}

*Se excluye una muerte por trauma del parto. ${ }^{* *}$ Se excluyen 5 muertes neonatales. ${ }^{* * *}$ Alguna morbilidad neonatal severa y muerte, patologías excluyentes. con SDR y displasia broncopulmonar, 12) y 13) neonatos con extensa bronconeumonía bilateral y muerte (Tabla II).

Microbiología del tracto genital inferior y del líquido amniótico. Una lista de microorganismos aislados de cervix/vagina y del líquido amniótico se muestra en la Tabla III. La frecuencia de IMCA fue $84,6 \%$ (11/13) en el grupo con RA y $50,0 \%$ $(27 / 54)$ en el grupo con RB $(p<0,05)$. Todas las pacientes con IMCA tuvieron infección cérvicovaginal. Infección cérvicovaginal sin infección intrauterina se presentó en $15,4 \%(2 / 13)$ en el grupo con RA y $31,5 \%$ (17/54) en el grupo con RB $(p>0,05)$. Pacientes sin infección (cérvix/vagina y líquido amniótico) no hubo en el grupo con RA y $18,5 \%(10 / 54)$ en el grupo con RB ( $p>0,05)$.

EI $S$. agalactiae se aisló más frecuentemente del líquido amniótico en el grupo con RA 23,1\% (3/13), que en el grupo con RB 3,7\% (2/54) $p<0,05$. No hubo diferencias significativas en los siguientes hallazgos microbiológicos del líquido amniótico entre los grupos: $U$. urealyticum la bacteria más frecuentemente aislada, seguida por $G$. vaginalis, $M$. hominis y $S$. viridans. Los microorga- 
Tabla III

\section{MICROORGANISMOS AISLADOS DE PACIENTES CON INFECCIÓN CERVICOVAGINAL E INVASIÓN MICROBIANA DE LA CAVIDAD AMNIÓTICA}

\begin{tabular}{|c|c|c|c|c|c|c|}
\hline \multirow[b]{2}{*}{ Microorganismos } & \multicolumn{2}{|c|}{ ICV } & \multicolumn{4}{|c|}{$I M C A$} \\
\hline & $\begin{array}{c}\text { Resultado* } \\
\text { adverso } \\
n=13\end{array}$ & $\begin{array}{c}\text { Resultado } \\
\text { bueno } \\
n=54\end{array}$ & Valor $p$ & $\begin{array}{c}\text { Resultado* } \\
\text { adverso } \\
n=13\end{array}$ & $\begin{array}{c}\text { Resultado } \\
\text { bueno } \\
n=54\end{array}$ & Valor $p$ \\
\hline Ureaplasma urealyticum & $8(61,5 \%)$ & $37(68,5 \%)$ & NS & $7(53,8 \%)$ & $23(42,6 \%)$ & NS \\
\hline Gardnerella vaginalis & $7(53,8 \%)$ & $21(38,9 \%)$ & NS & $3(23,1 \%)$ & $8(14,8 \%)$ & NS \\
\hline Mycoplasma hominis & $2(15,4 \%)$ & $7(13,0 \%)$ & NS & $1(7,7 \%)$ & $6(11,1 \%)$ & NS \\
\hline Streptococcus agalactiae & $4(30,8 \%)$ & $10(18,5 \%)$ & NS & $3(23,1 \%)$ & $2(3,7 \%)$ & $<0,05$ \\
\hline Streptococcus viridans & $2(15,4 \%)$ & $2(3,7 \%)$ & NS & $2(15,4 \%)$ & 0 & NS \\
\hline Enterococcus sp & 1 & $3(5,6 \%)$ & NS & $1(7,7 \%)$ & $1(1,9 \%)$ & NS \\
\hline Haemophilus influenzae & $2(15,4 \%)$ & $2(3,7 \%)$ & NS & $1(1,9 \%)$ & $3(5,6 \%)$ & NS \\
\hline Fusobacterium nucleatum & 0 & 0 & & $1(7,7 \%)$ & $1(1,9 \%)$ & NS \\
\hline Prevotella sp & 0 & 0 & & $1(7,7 \%)$ & $1(1,9 \%)$ & NS \\
\hline Streptococcus pneumoniae & 0 & 1 & NS & $1(7,7 \%)$ & $1(1,9 \%)$ & NS \\
\hline Proteus mirabilis & 1 & 0 & NS & $1(7,7 \%)$ & 0 & NS \\
\hline Peptostreptococcus sp & 0 & 0 & & $1(7,7 \%)$ & 0 & NS \\
\hline Neisseria gonorrhoeae & 0 & 1 & NS & 0 & $1(1,9 \%)$ & NS \\
\hline Staphylococcus coagulasa (-) & 0 & $3(5,6 \%)$ & NS & 0 & 0 & \\
\hline Escherichia coli & 0 & $3(5,6 \%)$ & NS & 0 & 0 & \\
\hline Candida albicans & 0 & $2(3,7 \%)$ & NS & 0 & 0 & \\
\hline Chlamydia trachomatis & 0 & $2(3,7 \%)$ & NS & 0 & 0 & \\
\hline Klebsiella pneumoniae & 0 & 1 & NS & 0 & 0 & \\
\hline Trichomona vaginalis & 0 & 1 & NS & 0 & 0 & \\
\hline
\end{tabular}

ICV: Infección cérvicovaginal. IMCA: Invasión microbiana de la cavidad amniótica.

IMCA: 84,6\% (11/13) resultado adverso; 50,0\% (27/54) resultado bueno $(p=0,03)$.

ICV sin IMCA: $15,4 \%(2 / 13)$ resultado adverso; $31,5 \%$ (17/54) resultado bueno ( $p=N S)$.

Sin IMCA, sin ICV: 0 resultado adverso; 18,5\% (10/54) resultado bueno ( $p=N S)$.

Vaginosis bacteriana: $61,5 \%(8 / 13)$ resultado adverso; $29,6 \%(16 / 54)$ resultado bueno $(p=0,03)$.

${ }^{*}$ Se excluye una muerte por trauma en el parto.

nismos más frecuentemente aislados de cervix/ vagina fueron $U$. urealyticum, $G$. vaginalis, $S$. agalactiae, $M$. hominis y $S$. viridans. C. trachomatis se aisló en 2 casos del grupo con RB. N. gonorrhoeae se aisló del líquido amniótico y cérvix en 1 caso del grupo con RB. La frecuencia de vaginosis bacteriana fue $61,5 \%(8 / 13)$ en el grupo con RA y
$29,6 \%(16 / 54)$ en el grupo con RB $(p<0,05)$ (Tabla III).

Hallazgos histológicos placentarios según grupos. Las placentas del grupo con RA tuvieron significativa más alta frecuencia de corioamnionitis histológica y funisitis que las del grupo con RB $(100 \%[13 / 13]$ vs $59,3 \%$ [32/54]; $p<0,01)$ y $(100 \%$

Tabla IV

HALLAZGOS HISTOLÓGICOS PLACENTARIOS

\begin{tabular}{lccc}
\hline Hallazgos histológicos & $\begin{array}{c}\text { Resultado adverso* } \\
n=13\end{array}$ & $\begin{array}{c}\text { Resultado bueno } \\
n=54\end{array}$ & Valor $p$ \\
\hline Corioamnionitis & $13(100 \%)$ & $32(59,3 \%)$ & $<0,01$ \\
Funisitis & $13(100 \%)$ & $24(44,4 \%)$ & $<0,001$ \\
Intervellitis & $5(38,5 \%)$ & $8(14,8 \%)$ & NS \\
Lesiones vasculares fetales & $2(15,4 \%)$ & $6(11,1 \%)$ & NS \\
Hiperplasia del trofoblasto & $2(15,4 \%)$ & $12(22,2 \%)$ & NS \\
Hallazgos negativos & 0 & $12(22,2 \%)$ & NS \\
\hline
\end{tabular}

*Se excluye una muerte por trauma en el parto. 
[13/13] vs $44,4 \%$ [24/54]; $p<0,001)$ respectivamente (Tabla IV).

Velocimetría Doppler. Los fetos que desarrollaron RA tuvieron la relación S/D de la AU expresada en percentil para la edad gestacional, significativamente más alta que los fetos con resultado bueno (RB) $(65,6 \pm 30,9$ vs $30,0 \pm 20,4 ; p<0,001)$, así como también más alta proporción de valores de la relación $\mathrm{S} / \mathrm{D}$ de la $\mathrm{AU}$ sobre el percentil 90 $(30,8 \%$ [4/13] vs $0 \%$ [0/54], respectivamente; $p<0,0001)$. No hubo diferencias en la relación $S / D$ de la AU en los grupos con y sin IMCA y con o sin funisitis (Tabla V). Los fetos con la relación $S / D$ de la $A U$ con percentil > 41 tuvieron significativo más alto riesgo de RA que fetos con la relación $S / D$ de la AU con percentil < 41 (odds ratio: 15,$77 ; 95 \% \mathrm{Cl}$ $2,73-118 ; p<0,001)$ con sensibilidad de $85 \%$, tasa de falso-positivo de $56 \%$, especificidad de $74 \%$ y falso-negativo de $5 \%$ (Figura 2). Tabla VI se muestran los valores de la relación S/D de la AU correspondiente a los percentiles críticos.

\section{DISCUSIÓN}

Por las consecuencias de la infección intrauterina (corioamnionitis clínica, corioamnionitis histológica y aumento de citoquinas intraamnióticas) y su relación con morbimortalidad neonatal aguda, daños neurológicos y enfermedad crónica pulmonar (3-5,7-10) es necesario disponer de un procedimiento destinado a pronosticar estos daños perinatales. En este estudio encontramos que en los casos de RPMPT antes de las 34 semanas de gestación, la relación $S / D$ de la $A U$, predice el resultado neonatal compuesto severo, definido como alguna morbilidad neonatal severa (síndrome de dificultad respiratoria, asfixia, sepsis, bronconeumonía, hemorragia intraventricular), se- cuelas (leucomalacia periventricular, displasia brocopulmonar) y muerte (19). Esta relación en cambio, no sirve para detectar IMCA y funisitis, a pesar que todos los casos con RA neonatal presentaron funisitis.

Se ha demostrado en varias publicaciones, el beneficio del Doppler para pronosticar el RA neonatal. La medición de la velocimetría de la AU, en fetos con retraso del crecimiento intrauterino predice complicaciones perinatales y admisión a la Unidad de Cuidado Intensivo $(13,14)$. En cambio esta relación en la RPMPT, no ha tenido la misma utilidad. No se ha logrado relacionar con IMCA, corioamnionitis clínica, inflamación placentaria o infección perinatal. Es posible que la correlación no se haya encontrado, porque estas características se analizaron individualmente (15-18).

En este estudio para mejorar la eficiencia del Doppler, la combinación de serias morbilidades neonatales resultó un método más apropiado que considerar estas patologías individualmente. La elevada frecuencia de RA neonatal encontrada (19\%) podría explicar la utilidad del Doppler en este trabajo. A su vez este RA dependería de la alta frecuencia, de IMCA (85\%) por Streptococcus agalactiae principalmente (23\%), de corioamnionitis histológica (100\%) y de funisitis (100\%) presentes en este estudio.

Aunque tradicionalmente el umbral de la anormalidad se considera la medición > del percentil 95, en nuestro trabajo con este percentil se detecta sólo el $23 \%$ de los casos con RA. En cambio cuando se usa la medición > del percentil 41, óptimo punto de corte de la curva relación S/D de la $\mathrm{AU}$, permite identificar el $85 \%$ de dichos fetos (11 de los 13 casos de esta serie) con tasa de falsos negativos de $5 \%$ y tasa de falsos positivos de $56 \%$. El resultado positivo falso puede mejorar-

Tabla V

RELACIÓN ENTRE ÍNDICE SÍSTOLE DIÁSTOLE DE LA ARTERIA UMBILICAL, IMCA, FUNISITIS Y MORBILIDAD GRAVE Y/O MUERTE, EXPRESADA EN PERCENTIL

\begin{tabular}{|c|c|c|c|c|c|c|c|c|c|}
\hline & $\begin{array}{c}\text { Con } \\
\text { IMCA } \\
n=39\end{array}$ & $\begin{array}{l}\operatorname{Sin} \\
\text { IMCA } \\
n=29\end{array}$ & $\begin{array}{l}\text { Valor } \\
p\end{array}$ & $\begin{array}{c}\text { Con } \\
\text { funisitis } \\
n=37\end{array}$ & $\begin{array}{c}\text { Sin } \\
\text { funisitis } \\
n=31\end{array}$ & $\begin{array}{l}\text { Valor } \\
p\end{array}$ & $\begin{array}{c}\text { Resultado } \\
\text { adverso } \\
n=13^{*}\end{array}$ & $\begin{array}{c}\text { Resultado } \\
\text { bueno } \\
n=54\end{array}$ & $\begin{array}{l}\text { Valor } \\
p\end{array}$ \\
\hline \multicolumn{10}{|l|}{$\begin{array}{l}\text { Arteria umbilical S/D } \\
\text { percentil }\end{array}$} \\
\hline Media (DS) & $40,5(30,7)$ & $32,1(19,3)$ & NS & $41,5(30,2)$ & $30,9(20,2)$ & NS & $65,6(30,9)$ & $30,0(20,4)$ & $<0,001$ \\
\hline Rango & $1-100$ & $2,5-79$ & & $1-100$ & $6,7-79$ & & $2-100$ & $1-79$ & \\
\hline $\begin{array}{l}\text { Arteria umbilical } S / D \\
\text { percentil }>95\end{array}$ & $4(10,3 \%)$ & 0 & NS & $4(10,8 \%)$ & 0 & NS & $4(30,8 \%)$ & 0 & $<0,0001$ \\
\hline
\end{tabular}

*Se excluye una muerte por trauma en el parto. 


\section{ROC Curve}

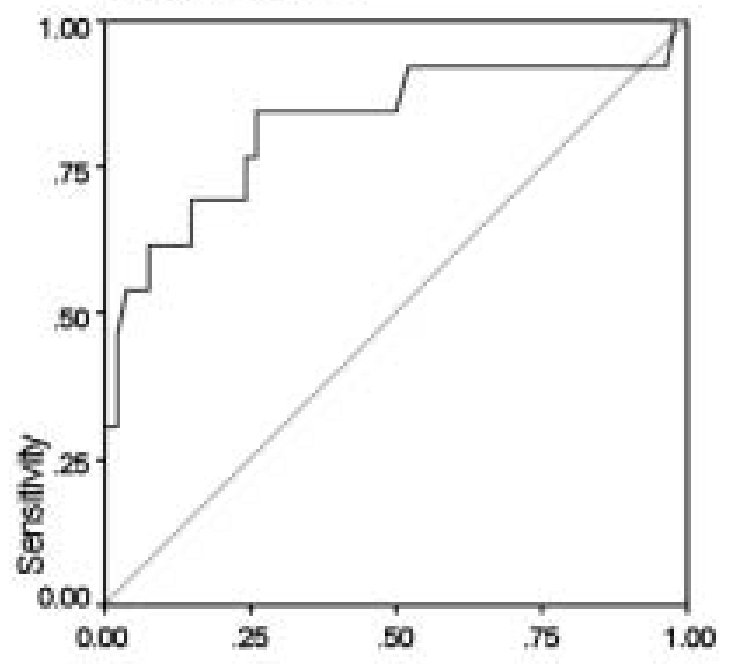

1 - Specificity

\section{Diagonal segments are produced by ties.}

Figura 2. Análisis de Curva Roc en pacientes con resultado neonatal adverso. Punto de corte en percentil 41; Sensibilidad 84,6\%, Especificidad 74,1\%, Falso-positivo $56 \%$, Falso negativo $4,8 \%$.

se con otras pruebas que miden bienestar fetal como el perfil biofísico (39). La alta especificificidad de $74 \%$ con bajo índice de falsos negativos nos permite optar con tranquilidad por una conducta expectante en el manejo de estos casos. En otras palabras cualquier valor de la relación $S / D$ de la $A U$ que se encuentre en embarazadas con RPMPT por sobre el percentil 41 es potencialmente peligroso para el feto. Por el contrario si esta relación se mantiene por debajo del percentil 41 tenemos el $74 \%$ de seguridad que el producto no tendrá un resultado adverso.

Es posible que los resultados adversos de esta serie también estén influenciados por la menor edad gestación al momento de la admisión (27,1 vs 30,9 semanas) y el menor peso de nacimiento (1.208 vs 2.010 gramos) que tuvo este grupo en relación al de buen resultado neonatal. Sin embargo también es probable que los embarazos de menor edad gestacional son mas vulnerables a bacterias de gran virulencia para los neonatos como Streptococcus agalactiae, Haemophilus influenzae, Fusobacterium nucleatum y Streptococcus pneumoniae bacterias que preferentemente se aislaron del líquido amniótico en estos casos (40).
Tabla VI

COMPARACIÓN VALORES CRÍTICOS DE PERCENTILES DE LA MEDICIÓN S/D DE ARTERIA UMBILICAL POR EDAD GESTACIONAL

\begin{tabular}{lcc}
\hline & \multicolumn{2}{c}{$S / D A U$} \\
EG semanas & Percentil 41 & Percentil 95 \\
\hline 24 & 3,30 & 4,50 \\
25 & 3,21 & 4,39 \\
26 & 3,14 & 4,27 \\
27 & 3,05 & 4,15 \\
28 & 2,97 & 4,03 \\
29 & 2,88 & 3,92 \\
30 & 2,81 & 3,80 \\
31 & 2,72 & 3,68 \\
32 & 2,64 & 3,57 \\
33 & 2,56 & 3,45 \\
34 & 2,48 & 3,33 \\
35 & 2,40 & 3,22 \\
\hline
\end{tabular}

EG: edad gestacional. S/D: sístole/diástole. AU: arteria umbilical.

Con el Doppler de la AU no es posible detectar IMCA y funisitis. Una explicación de este hallazgo es que no todas las infecciones intraamnióticas que causan funisitis producirán RA en el neonato. Probablemente el buen resultado en estas situaciones se deba a carga bacteriana variable, a la diferente inmunidad fetal que disminuye con la edad gestacional, a la distinta virulencia bacteriana y especialmente a la acción beneficiosa de los antimicrobianos (30, 41-43).

\section{CONCLUSIÓN}

Nuestro estudio permite concluir que en embarazadas con rotura prematura de membranas de pretérmino y funisitis, el Doppler de la AU puede discriminar entre los fetos que tendrán resultado adverso y aquéllos con buen resultado neonatal.

\section{BIBLIOGRAFÍA}

1. Meis PJ, Ernest JM, Moore ML. Causes of low birth weight births in public and private patients. Am J Obstet Gynecol 1987; 156: 1165-8.

2. Gibbs RS, Blanco JD. Premature rupture of the membranes. Obstet Gynecol 1982; 60: 671-9.

3. Romero R, Ghidini A, Mazor M, Behnke E. Microbial invasion of the amniotic cavity in premature rupture of membranes. Clin Obstet Ginecol 1991; 4: 769-78.

4. Romero R, Quintero R, Oyarzún E, Wu YK, Sabo V, Mazor M, et al. Intraamniotic infection and the onset of labor in preterm premature rupture of the membranes. Am J Obstet Gynecol 1988; 159: 6616. 
5. Gómez R, Ghezzi R, Romero R, Muñoz H, Tolosa J, Rojas Iván. Premature labor and intra-amniotic infection. Clin Perinatol 1995; 22: 281-342.

6. Ovalle A, Martínez MA, Giglio MS, Poblete P, Fuentes A, Villablanca E. Microbiología aislada en la rotura prematura de membranas de pretérmino. Relación con morbilidad infecciosa materna neonatal e intervalo rotura de membranas-parto. Rev Chil Obstet Ginecol 1995; 60: 252-62.

7. Gómez R, Romero R, Ghezzi F, Yoon BH, Mazor M, Berry SM. The fetal inflammatory response syndrome. Am J Obstet Gynecol 1998; 179(1): 194202.

8. Hagberg H, Wennerholm UB, Savman K. Sequelae of chorioamnionitis. Curr Opin Infect Dis 2002; 15(3): 301-6.

9. Vigneswaran R. Infection and preterm birth: evidence of a common causal relationship with bronchopulmonary dysplasia and cerebral palsy. J Paediatr Child Health 2000; 36(4): 293-6.

10. Schendel DE, Schuchat A, Thorsen P. Public health issues related to infection in pregnancy and cerebral palsy. Ment Retard Dev Disabil Res Rev 2002; 8(1): 39-45.

11. Ovalle A, Martínez MA, Kakarieka ME, Giglio MS, Fuentes A, Gómez R, et al. Hallazgos histopatológicos de la placenta en la rotura prematura de membranas de pretérmino. Relación con la microbiología aislada. Rev Méd Chile 1998; 126: 911-918.

12. Yoon BH, Romero R, Park JS, Kim CJ, Kim SH, Choi $\mathrm{JH}$, et al. Fetal exposure to an intra-amniotic inflammation and development of cerebral palsy at the age of three years. Am J Obstet Gynecol 2000; 182: 675-81.

13. Vergani P, Andreotti C, Roncaglia N, Zani G, Pozzi E, Pezzullo JC, et al. Doppler predictors of adverse neonatal outcome in the growth restricted fetus at 34 weeks' gestation or beyond. Am J Obstet Gynecol 2003; 189(4): 1007-11.

14. Soregaroli M, Bonera R, Danti L, Dinolfo D, Taddei F, Valcamonico A, et al. Prognostic role of umbilical artery Doppler velocimetry in growth-restricted fetuses. J Matern Fetal Neonatal Med 2002; 11(3): 199-203.

15. Abramowicz JS, Sherer DM, Warsof SL, Levy DL. Fetoplacental and uteroplacental Doppler blood flow velocity analysis in premature rupture of membranes. Am J Perinatol 1992; 9(5-6): 353-6.

16. Ghidini A, Salafia CM, Kirn V, Doria V, Spong CY. Biophysical profile in predicting acute ascending infection in preterm rupture of membranes before 32 weeks. Obstet Gynecol 2000; 96(2): 201-6.

17. Leo MV, Skurnick JH, Ganesh VV, Adhate A, Apuzzio JJ. Clinical chorioamnionitis is not predicted by umbilical artery Doppler velocimetry in patients with premature rupture of membranes. Obstet Gynecol 1992; 79(6): 916-8.

18. Carroll SG, Papaioannou S, Nicolaides KH. Doppler studies of the placental and fetal circulation in pregnancies with preterm prelabor amniorrhexis. Ultrasound Obstet Gynecol 1995; 5(3): 184-8.

19. Mercer BM, Miodovnik M, Thurnau GR, Goldenberg $\mathrm{RL}$, Das AF, Ramsey RD, et al. Antibiotic therapy for reduction of infant morbidity after preterm premature rupture of the membranes. A randomized controlled trial. National Institute of Child Health and Human Development Maternal-Fetal Medicine Units Network. JAMA 1997; 278: 989-95.

20. Juez G, Lucero E, Ventura-Junca P, Tapia JL, Winter A. Crecimiento intrauterino en recién nacidos chilenos de clase media. Rev Chil Pediatr 1989; 60: 198202.

21. Taylor-Robinson D, Furr PM. Recovery and identification of genital tract Mycoplasmas. Israel J Med Sci 1981; 17: 648-53.

22. Claas HC, Melchers WJ, De Bruijn IH, De Graaf M, Van Dijk WC, Lindeman J, et al. Detection of Chlamydia trachomatis in clinical specimens by the polymerase chain reaction. Eur J Clin Microbiol \& Infect Dis 1990; 9: 864-8.

23. Wang E, Richardson H. A rapid method for detection of group B streptococcal colonization: testing at the bedside. Obstet Gynecol 1990; 76: 882-5.

24. Odds FC, Bernaerts R. CHROMagar Candida, a new differential isolation medium for presumptive identification of clinically important Candida species. J Clin Microbiol 1994; 32: 1923-9.

25. Villagra E, Martínez MA, Ovalle A. Flora microbiana genital en una población de alto riesgo obstétrico. Rev Chil Obstet Ginecol 1994; 59: 32-38.

26. Murray PR, Baron EJ, Jorgensen JH, Pfaller MA, Yolken RH (Eds). Manual of Clinical Microbiology. 7th Ed. American Society for Microbiology, Washington DC, 1999.

27. Nugent R, Krohn M, Hillier S. Reliability of diagnosing bacterial vaginosis is improved by standardized method of Gram stain interpretation. J Clin Microbiol 1991; 29: 297-301.

28. Romero R, Mazor M, Morrotti R, Avila C, Oyarzún $E$, Insunza A, et al. Microbial invasion of the amniotic cavity in spontaneous rupture of membranes at term. Am J Obstet Gynecol 1992; 166: 129-33.

29. McGregor JA, French JI, Jones W, Parker R, Patterson E, Draper D. Association of cervicovaginal infections with increased vaginal fluid phospholipase A2 activity. Am J Obstet Gynecol 1992; 167: 158894.

30. Ovalle A, Martinez MA, Gomez R, Rubio R, Fuentes A, Valderrama O. Antibiotic therapy in patients with preterm premature of membranes: a prospective, randomized, placebo controlled study with microbiologic assessment of the amniotic cavity and lower genital tract. Prenat Neonat Med 1997; 2: 213-22.

31. Gibbs RS, Castillo MS, Rogers PJ. Management of acute chorioamnionitis. Am J Obstet Gynecol 1980; 136: 109-13.

32. Oyarzún E, Gormaz G. Ultrasonografía en Obstetri- 
cia.. Capítulo 6: Doppler y Vigilancia Antenatal. pp 82-110. Editorial Mediterráneo, 2003.

33. Papile LA, Burstein J, Burstein R, Koffler $\mathrm{H}$. Incidence and evolution of subependymal and intraventricular hemorrhage: a study of infants with birth weights less than 1,500 g. J Pediatr 1978; 92(4): 529-34.

34. Bernischke K, Kaufman P. Pathology of the human placenta. Chapter: Histopathological Approach to Villous Alteration, pp 419-434. Chapter: Infectious diseases, pp 591-659. New York NY: Springer Verlag NY, Inc; 2001.

35. Perrin VDK. Pathology of the Placenta. Chapter: Placenta as a Reflection of maternal disease, pp 57-70. New York, Churchill Livingstone Inc; 1984.

36. Fox H. Pathology of the Placenta. Chapter: Histological Abnormalities of the Placenta, pp 149 197. Philadelfia, Pa: WB Saunders Co; 1978.

37. Keeling JW. Fetal and Neonatal Pathology. Chapter: Placenta and Umbilical Cord, pp 45-67. SprigerVerlag. Berlin Heidelgerg, 1987.

38. Van Hoeven $\mathrm{KH}$, Anyaegbunam A, Hochster $\mathrm{H}$, Whitty JE, Distant J, Crawford C, et al. Clinical significance of increasing histologic severity of acute inflammation in the fetal membranes and umbilical cord. Pediat Pathol 1996; 16: 731-44.

39. Fleming AD, Salafia CM, Vintzileos AM, Rodis JF,
Campbell WA, Bantham KF. The relationships among umbilical artery velocimetry, fetal biophysical profile, and placental inflammation in preterm premature rupture of the membranes. Am J Obstet Gynecol 1991; 164(1 Pt 1): 38-41.

40. Ovalle A, Gómez R, Martínez MA, Kakarieka E, Fuentes A, Aspillaga C, et al. Invasión microbiana de la cavidad amniótica en la rotura de membranas de pretérmino. Resultados maternoneonatales y patología placentaria según microorganismo aislado. Rev Med Chile 2005; 133: 55-61.

41. Mercer BM, Miodovnik M, Thurnau GR, Goldenberg $\mathrm{RL}$, Das AF, Ramsey RD, et al. Antibiotic therapy for reduction of infant morbidity after preterm premature rupture of the membranes. A randomized controlled trial. National Institute of Child Health and Human Development Maternal-Fetal Medicine Units Network. JAMA 1997; 278: 989-95.

42. Kenyon S, Boulvain M, Neilson J. Antibiotics for preterm rupture of membranes. Cochrane Database Syst Rev 2003; (2): CD001058.

43. Kenyon S, Taylor DJ, Tarnow-Mordi WO; ORACLE Collaborative Group. ORACLE-antibiotics for preterm prelabour rupture of the membranes: short-term and long-term outcomes. Acta Paediatr Suppl 2002; 91(437): 12-5. 